\title{
ALGEBRAIC IDEALS OF SEMIPRIME BANACH ALGEBRAS
}

\section{by S. GIOTOPOULOS and M. ROUMELIOTIS}

(Received 4 June, 1990)

Abstract. If $A$ is a semiprime Banach algebra, $\operatorname{soc} A, \operatorname{rad} A$ the socle and radical of $A$, then $\overline{\operatorname{soc} A} \cap \operatorname{rad} A=(0)$. This elementary result enables us to prove some results concerning algebraic ideal and algebraic elements modulo the socle of $A$. We also deduce several conditions for $A$ equivalent to the condition $\operatorname{dim} A<+\infty$.

Introduction. The class of semiprime Banach algebras is the class of algebras to which one might reasonably expect some theorems on semisimple Banach algebras to extend. However, there are results on semisimple Banach algebras that do not hold for semiprime ones; e.g. the automatic continuity of the functional calculus in commutative algebras fails to extend to semiprime algebras [3].

In this paper we prove some results concerning algebraic ideals and algebraic elements modulo the socle of a semiprime Banach algebra.

Our main results may be stated as follows. socle.

(1) The largest biideal of algebraic elements of a semiprime Banach algebra $A$ is its

(2) If $A$ is a semiprime Banach algebra with unit, $x \in A$ with $p(x) \in \operatorname{soc}(A)$ for some polynomial $p$, then $\exists w \in A$ such that $p(w)=0$ and $x-w \in \operatorname{soc}(A)$.

Result (1) extends Theorem 3.2 in [12] and it provides a characterization of the socle of $A$ and result (2) extends Theorem 12 in [5]. Notice that our proof of result (2) is simpler than the proof given in [5].

We also deduce several equivalent conditions which force the algebra to be finite dimensional.

In general notation and terminology used is as in [7]. In particular we write $\operatorname{soc} A=\{0\}$ if the algebra $A$ has no minimal idempotents.

All the algebras and linear spaces considered are over the complex field.

Lemma 1. Let $A$ be a Banach algebra with unit. If $x$ is an algebraic element in the radical of $A$, then $x$ is nilpotent.

Proof. Since $x$ is algebraic there exists a polynomial $p$ such that $p(x)=0$. The polynomial $p$ is of the form $p(\lambda)=\lambda^{n} q(\lambda)$ and the polynomial $q$ has non zero constant term, $\beta_{0}$ say. Then $q(x) \notin \operatorname{rad} A$ and by the spectral mapping theorem $\sigma(q(x))=$ $q(\sigma(x))=\{q(0)\}=\left\{\beta_{0}\right\} \neq\{0\}$. Hence $0 \notin \sigma(q(x))$ and therefore $q(x)$ is invertible, so that $0=0 . q(x)^{-1}=x^{n} q(x) \cdot q(x)^{-1}=x^{n}$.

REMARK 2. An argument similar to that in the proof of Lemma 1 shows that if $x$ is a quasinilpotent algebraic element which lies in a proper ideal $I$ of $A$ then $x$ is nilpotent.

An immediate consequence of Lemma 1 above is the following well-known result.

Proposition 3. If $A$ is a finite dimensional semiprime (Banach) algebra, then $A$ is semisimple.

The following gives an example of a non-radical semiprime Banach algebra $A$ with $\operatorname{rad} A \neq\{0\}$.

Glasgow Math. J. 33 (1991) 359-363. 
ExAmple 1. Let $F$ be the free algebra on two symbols $u, v$ and let $\left\{w_{n}\right\}$ be the standard enumeration of the words given by $u, v, u^{2}, u v, v u, v^{2}, u^{3}, u^{2} v, \ldots$ and let $\lambda_{n}$ be the length of the word $w_{n}$. Let $A$ be the algebra of all infinite series $x=\sum \alpha_{n} w_{n}$, where

$$
\|x\|=\sum \frac{\left|\alpha_{n}\right|}{\left(\lambda_{n}\right) !}<+\infty .
$$

Then $A$ is a non-commutative semiprime radical Banach algebra ([7, pp. 254-255]).

Let $A_{1}=A+\mathbb{C}$, the unitization of $A$ over $\mathbb{C}\left(\left[7\right.\right.$, Def. 1, p. 15]). It is clear that $A_{1}$ is semiprime since $A$ has no divisors of zero, and $A_{1}$ is not radical since $(0, \lambda) \notin \operatorname{rad} A_{1}$ for $\lambda \in \mathbb{C}-\{0\}$. But $\operatorname{rad} A_{1} \neq\{0\}$. In fact if $d$ is a non zero element of $A$ then $(d, 0) \in \operatorname{rad} A_{1}$.

Example 2. Let $F, A$ be as in Example 1 and $B$ the algebra of all infinite series $x=\sum \alpha_{n} w_{n}$, where

$$
\|x\|^{\prime}=\sum\left|\alpha_{n}\right|<+\infty .
$$

Then $B$ is a non-commutative semisimple Banach algebra. Let $D=\{(a, b): a \in A, b \in B\}$, where

$$
\begin{aligned}
(a, b)+\left(a^{\prime}, b^{\prime}\right) & =\left(a+a^{\prime}, b+b^{\prime}\right), \\
\lambda .(a, b) & =(\lambda a, \lambda b), \\
\left(a, a^{\prime}\right) .\left(b, b^{\prime}\right) & =\left(a b+b^{\prime} a+a^{\prime} b, a^{\prime} b^{\prime}\right),
\end{aligned}
$$

for all $a, a^{\prime} \in A, b, b^{\prime} \in B, \lambda \in \mathbb{C}$ and

$$
\|(a, b)\|=\|a\|+\|b\|^{\prime} .
$$

Then $D$ is a non-commutative semi-prime Banach algebra which is not radical (since $(0, b) \notin \operatorname{rad} D)$. But $\operatorname{rad} D \neq\{0\}$ since $(a, 0) \in \operatorname{rad} D$.

LemMa 4. Let $A$ be a semiprime Banach algebra with unit and let $M$ be a biideal of algebraic elements of $A$.

(i) $\bar{M} \cap \operatorname{rad} A=\{0\} ;$ in particular $\overline{\operatorname{soc} A} \cap \operatorname{rad} A=\{0\}$.

(ii) $\bar{M}$ is a semisimple Banach algebra.

(iii) If $f$ is an idempotent element in $M$, then $f A f$ is a finite dimensional semisimple Banach algebra with unit $f$ and $f \in \operatorname{soc} A$.

(iv) For every nonzero element $m \in M$ the principal left (right) ideal Am ( $m A)$ contains minimal idempotents and every orthogonal set of minimal idempotents in Am $(m A)$ is finite.

(v) For every element $m \in M$ there exists an idempotent $f$ in $\operatorname{soc} A$ such that $m=m f$, and $f \in A m$.

(vi) $M \subset \operatorname{soc} A$.

Proof. (i) From Lemma 1 it follows that the biideal $M \cap \operatorname{rad} A$ is nil and from [9] (or Corollary 5 of $[7$, p. 254]) it follows that $M \cap \operatorname{rad} A=\{0\}$. Hence $N \subset \operatorname{lan} \operatorname{rad} A$. Let $x \in \bar{M} \cap \operatorname{rad} A$. Then there exists a sequence $\left(x_{n}\right)$ in $M \subset \operatorname{lan} \operatorname{rad} A$ with $x_{n} \rightarrow x$. Hence $0=x_{n} x \rightarrow x^{2}$ and therefore $x^{2}=0$. This implies that $\bar{M} \cap \operatorname{rad} A$ is nil and so $\bar{M} \cap \operatorname{rad} A=$ $\{0\}$ as above. 
Suppose now that $x \in \operatorname{soc} A$ and $x=\alpha_{1} e_{1}+\alpha_{2} e_{2}+\ldots+\alpha_{n} e_{n}$, where $e_{1}, e_{2}, \ldots, e_{n}$ are minimal idempotents of $A$ and $\alpha_{1}, \alpha_{2}, \ldots, \alpha_{n} \in A$. Then $x A x \subset \sum_{i, j=1}^{n} \alpha_{i} e_{i} A \alpha_{j} e_{j}$ has finite dimension by Theorem 6 (iii) of [7, p. 158].

Hence $x$ is algebraic. Since $\operatorname{soc} A$ is a biideal of algebraic elements of $A$ we have $\overline{\operatorname{soc} A} \cap \operatorname{rad} A=\{0\}$. $\{0\}$.

(ii) From Corollary 20 of $[7$, p. 126] and (i) above we see that $\operatorname{rad} \bar{M}=\bar{M} \cap \operatorname{rad} A=$

(iii) It is clear that $\{0\} \neq f A f=f \bar{M} f \subset M$ and $f \bar{M} f$ is semisimple from BA.2.7 of [6, p. 104]. Therefore $f A f$ is semisimple. Now, from the spectral mapping theorem it follows that every element of $M$ (and therefore every element of $f \bar{M} f=f A f$ ) has finite spectrum. Kaplansky's theorem ([11]) asserts that $f \bar{M} f$ is finite dimensional and thus $f \in \operatorname{soc}(\bar{M})=$ $\bar{M} \cap \operatorname{soc} A$ (from Theorem 7.2 of [1]).

(iv) Let $m$ be a non-zero element in $M$. From (i) it follows that there exists $u \in A$ such that um is not quasinilpotent. It is clear that the spectrum of $u m$ is finite.

We denote by $B$ the commutative Banach algebra generated by um. Since um is algebraic, $B$ is a finite-dimensional algebra which is not radical and which therefore possesses a non-zero idempotent $f$, from the well-known Artin-Wedderburn theory. From (iii) $f \in \operatorname{soc} A$; therefore $A m \cap \operatorname{soc} A \neq\{0\}$, which implies that $A m$ contains minimal idempotents.

An argument analogous to that in the proof of Lemma 3.1(iv) of [12] proves that every orthogonal set of minimal idempotents in $\operatorname{Am}(m A)$ is finite.

In fact, if $\left\{e_{1}, e_{2}, \ldots\right\}$ is an infinite set of pairwise orthogonal minimal idempotents in $A m$ then we can choose a sequence $\left(a_{n}\right)_{n \geq 1}$ in $A$ and a sequence $\left(\lambda_{n}\right)_{n \geq 1}$ in $\mathbb{C}$ such that $e_{n}=a_{n} m$ and $\left|\lambda_{n}\right|<2^{-n} \cdot\left\|a_{n}\right\|^{-1}$ for each $n=1,2 \ldots$ Then $\sum_{n=1}^{\infty} \lambda_{n} a_{n}$ converges to an element $a$ (say) in $A$ and $a m=\sum_{n=1}^{\infty} \lambda_{n} a_{n} m=\sum_{n=1}^{\infty} \lambda_{n} e_{n}$. Since $e_{n} a m=\lambda_{n} e_{n}$ or $e_{n}\left(\lambda_{n}-a m\right)=$ 0 for each $n$, we have that $\lambda_{n}-a m$ is not invertible and therefore $\lambda_{n} \in \sigma(a m)$ for each $n=1,2, \ldots$; this contradicts the finiteness of the spectrum $\sigma(\mathrm{am})$.

(v) Let $m \in M$. If $m=0$ we take $f=0$. Suppose $m \neq 0$. From (iv) above there exists a finite maximal set $E=\left\{e_{1}, e_{2}, \ldots, e_{n}\right\}$ of minimal (pairwise orthogonal) idempotents in $A m$. Note that $m e_{i} \neq 0$ for some $1 \leq \mathrm{i} \leq \mathrm{n}$. Let $f=e_{1}+e_{2}+\ldots+e_{n}$. It is clear that $f^{2}=f \in \operatorname{soc} A \cap A m$.

We claim that $m=m f$. For if $m-m f \neq 0$, then the principal right ideal $(m-m f) A$ contains a minimal idempotent, $e$ say. Then $e=(m-m f) u$ for some $u \in A$. Define $e_{n+1}=(1-f)$ uem $(1-f)$. We can now easily verify that

$$
e_{n+1}^{2}=e_{n+1}=(1-f) u e m-(1-f) u e m f \in A m+A m \subset A m
$$

and $0=e_{n+1} \cdot e_{j}=e_{j} \cdot e_{n+1}$ for $j=1,2, \ldots, n$; i.e. $e_{n+1}$ is a minimal idempotent in $A m$ orthogonal to all the $e_{j}^{\prime}$ s, contradicting the maximality of $E$.

(vi) This follows immediately from (v) above.

Now Theorem 5 below follows immediately. 
THEOREM 5. If $A$ is a semiprime Banach algebra with unit, then the socle is the largest biideal which lies in the algebraic elements of $A$.

We also obtain the following characterizations of the socle of the algebra $A$ which extend theorem 7.2 of [1].

Corollary 6. If $A$ is a semiprime Banach algebra with unit, $\Sigma=\{x \in A: \operatorname{dim}(x A x)<$ $+\infty\}$ and $F=\{x \in \Sigma: \operatorname{dim}(x A u)<+\infty \forall u \in \Sigma\}$, then $\operatorname{soc} A=F=\Sigma$.

Proof. Since $F$ is a biideal of algebraic elements of $A$ we have $F \subset \operatorname{soc} A \subset \Sigma$. Let $\mathrm{x} \in \Sigma$ and denote by $\langle x\rangle$ the biideal of $A$ generated by $x$. A typical element of $\langle x\rangle$ is of the form $y=\lambda x+a x+x b+\sum_{i=1}^{k} a_{i} x b_{i}$. It is clear that $\operatorname{dim}(y A y)<+\infty$ and therefore $\langle x\rangle \subset \Sigma$. From Theorem 5 it follows that $\langle x\rangle \subset \operatorname{soc} A$. Hence $\Sigma \subset \operatorname{soc} A$ and therefore $\Sigma=\operatorname{soc} A$. It is now obvious that $F=\operatorname{soc} A$ too.

An easy consequence of known results is the following theorem.

THEOREM 7. Let $A$ be a semiprime algebra with unit. The following conditions are equivalent:

(i) A has finite dimension,

(ii) $A$ is algebraic,

(iii) $A=\operatorname{soc} A$,

(iv) $A$ is locally finite,

(v) every singly-generated subalgebra of $A$ is finite dimensional.

Proof. An element $x$ of $A$ is algebraic if and only if it belongs to a finite dimensional subalgebra. Hence (ii) $\Leftrightarrow$ (v).

Now if $A$ is semiprime and finite dimensional, then $A$ is semisimple and finite dimensional and therefore $A=\operatorname{soc} A$. Hence (i) $\Rightarrow$ (iii). The conclusion (iii) $\Rightarrow$ (i) is a result of Tullo [13] and the equivalence (iv) $\Leftrightarrow(v) \Leftrightarrow$ (i) follows from the second theorem of $[\mathbf{1 0}]$.

The equivalence (i) $\Leftrightarrow$ (iii) has also been obtained jointly by L. Dalla and N. Katseli by a different approach using the concept of "single" element [8].

The following is related to Proposition 2.8 of [2].

LeMma 8. Let $A$ be a semiprime Banach algebra with unit. If $x \in A, x^{n} \in \operatorname{soc} A$ and $x=$ exe, where $e=e^{2} \in A$, then there exists $w \in A$ with $w=e w e, w^{n}=0$ and $x-w \in \operatorname{soc} A$.

Proof We may assume that $x \notin \operatorname{soc} A$, for if $x \in \operatorname{soc} A$ we take $w=0$.

Case 1. $\sigma(x)=\{0\}$. Then $x$ is nilpotent, say $x^{N}=0$. Let $m$ be the smallest positive integer for which there exist $y \in A$ with $x-y \in \operatorname{soc} A, y=e y e$ and $y^{m}=0$. Since $N$ is such an integer, this $m$ exists. If $m \leqslant n$, then we take $w=y$ and the result is proved. Suppose otherwise; then $m-1 \geq n$, and so $x^{m-1} \in \operatorname{soc} A$; also $y^{m-1} \in \operatorname{soc} A$. Therefore there exist $f=f^{2} \in \operatorname{soc} A$ with $y^{m-1}=y^{m-1} f, f \in A y^{m-1}$ and $f y \in A y^{m}=\{0\}$, by Lemma $4(\mathrm{v})$. Put $w=y(1-f) e$. Then $w^{m-1}=0, w=e w e$, and $x-w \in \operatorname{soc} A$, since $x-y(1-f) \in \operatorname{soc} A$. This contradicts the supposed minimality of $m$. 
Case 2. $\sigma(x) \neq\{0\}$. Since $x^{n} \in \operatorname{soc} A$ and $0 \in \sigma(x)$ there exists a polynomial $p$ with no constant term such that $p\left(x^{n}\right)=0$; let

$$
p\left(x^{n}\right)=x^{k n}\left(1+\lambda_{1} x^{n}+\ldots+\lambda_{m} \cdot x^{m n}\right) .
$$

Put $v=x\left(1+\lambda_{1} x^{n}+\ldots+\lambda_{m} \cdot x^{m n}\right)$. Now if $e=e^{2} \in A$ and $x=e x e$, then $x-v \in \operatorname{soc} A$, $v=e v e, v^{n} \in \operatorname{soc} A$ and $v^{k n}=0$. Therefore Case 1 applies to give an element $w=e w e \in A$ with $w^{n}=0, v-w \in \operatorname{soc} A$ and $\operatorname{so} x-w \in \operatorname{soc} A$.

It is clear that Lemma 8 is true in the particular case where $e$ is the unit of $A$.

We are indebted to the referee for shortening our original proof of Case 1 of Lemma 8.

Theorem 9. Let $A$ be a semiprime Banach algebra with unit and let $x \in A$ with $p(x) \in \operatorname{soc} A$ for some polynomial $p$. Then there exists an element $w \in A$ with $p(w)=0$ and $x-w \in \operatorname{soc} A$.

Proof. This follows immediately from Lemma 1 of Barnes' paper [5]: our Lemma 8 says that Barnes' condition $H_{N}$ holds for all $N$ with $K=\operatorname{soc} A$; hypothesis (2) of Barnes' Lemma 1 follows from the fact that the spectrum of $x$ is finite. This concludes the proof.

\section{REFERENCES}

1. J. C. Alexander, Compact Banach algebras, Proc. London Math. Soc. (3) 18 (1968), 1-18.

2. C. Akemann and G. K. Pedersen, Ideal perturbations in $\mathrm{C}^{*}$-algebras, Math. Scand. 41 (1977), 117-139.

3. G. R. Allan, Embedding the algebra of formal power series in a Banach algebra, Proc. London Math. Soc. (3) 25 (1972), 329-340.

4. B. Barnes, On the existence of minimal ideals in a Banach algebra, Trans. Amer. Math. Soc. 133 (1968), 511-517.

5. B. Barnes, Algebraic elements of a Banach algebra modulo an ideal, Pacific J. Math. 117 (1985), 219-231.

6. B. Barnes, G. Murphy, M. Smyth and T. West, Riesz and Fredholm theory in Banach algebras, Research Notes in Mathematics 67 (Pitman, London, 1982)).

7. F. Bonsall and J. Duncan, Complete normed algebras, (Springer-Verlag, 1973).

8. Leoni Dalla, S. Giotopoulos and Nelli Katseli, The socle and finite dimensionality of a semiprime Banach algebra, Studia Math. 92 (1989), 201-204.

9. P. G. Dixon, Semiprime Banach algebras, J. London Math. Soc. (2) 6 (1973), 676-678.

10. P. G. Dixon, Locally finite Banach algebras, J. London Math. Soc. (2) 8 (1974), 325-328.

11. I. Kaplansky, Ring isomorphisms of Banach algebras, Canad. J. Math. 6 (1954), 374-381.

12. M. R. F. Smyth, Riesz theory in Banach algebras, Math. Z. 145 (1975), 145-155.

13. A. W. Tullo, Conditions on Banach algebras which imply finite dimensionality. Proc. Edinburgh Math. Soc. (2) 20 (1976), 1-5.

\section{Department of Mathematics}

ATHENS UNIVERSITY

Panepistemiopolis

15784 ATHENS

GREECE 